\title{
ON CERTAIN POLAR CURVES WITH THEIR APPLICATION TO THE LOCATION OF THE ROOTS OF THE DERIVATIVES OF A RATIONAL FUNCTION*
}

BY

\author{
B. Z. LINFIELD
}

\section{INTRODUCTION}

The question asked by Professor Maxime Bôchert in a discussion concerning certain theorems on the roots of the derivative of a polynomial, "Could not the first of these propositions be brought into connection with the focal properties of the higher plane curves?" had been answered earlier by M. van den Berg‡ with the following theorem:§ Les points racines de la dérivée d'une équation à $n$ racines différentes sont les $n-1$ foyers d'une courbe de $(n-1)$ e classe qui touche dans leurs milieux les $\frac{1}{2} n(n-1)$ côtes du $n$-agone complet déterminé pav l'équation originale. L'équation originale ayant des racines multiples, la droite joignant un point-racine $m^{p l e} \grave{a}$ un point-racine $p^{\text {ple }}$ est divisée à raison de $m$ ì $p$ par le point de contact. An independent proof of this theorem was given also by P. J. Heawood for the polynomial with simple roots, and the extension to the case of multiple roots was indicated\|. M. Fujiwara $\mathbb{T}$ generalized van den Berg's theorem as follows: Die Wurzelpunkte von $f^{k}(x)=0$ sind die Brennpunkte der kten polaren Kurve $(n-k$ ter Klasse) der Kurve nter Klasse, welche aus den $n$ Wurzelpunkte von $f(x)=0$ besteht, in bezug auf die unendlich-ferne Gerade. Apparently the last writer dealt with a polynomial of simple roots, and he therefore failed to note the relationship of his $k$ th polar

\footnotetext{
- Presented to the Society October 28, 1922.

t Annals of Mathematics, vol. 7 (1892), p. 70.

$\ddagger$ Nieuw Archief voor Wiskunde, vol. 15 (1888), p. 190 .

$\S$ The theorem is stated as quoted by M. Jan de Vries, Rendiconti del Circolo Matematico di Palermo, vol. 5 (1891), p. 290.

\|Quarterly Journal of Mathematics, vol. 38 (1907), p. 80.

The writer in ignorance of the contents of these papers and in the belief that Bôcher's question was unanswered, proved this theorem for the rational function of multiple roots and poles, Bulletin of the American Mathematical Society, vol.27 (1920), p. 17. He is indebted to Professor A. J. Kempner and to Dr. J. L. Walsh for the references to van den Berg and Heawood. The investigation was undertaken at the suggestion of Professor W. H. Echols of the University of Virginia to whom the writer feels greatly indebted for encouragement and inspiration.
}

đT Tôhôku Mathematical Journal, vol. 9 (1916), p. 108. 
curve to the original polynomial of the roots of $f(x)=0$, as van den Berg has done in the case of the first derivative of a polynomial, and as the writer will do for the case of the $r$ th derivative of a rational function of multiple zeros and poles. Some theorems concerning the properties of these curves will then be taken up, from which the foci will be localized to limited areas, and applications will be made to particular functions where it will be shown that the curves can be traced with ease.

PART I

\section{NOTATION AND PRELIMINARY LEMMAS}

The operation of polarization with respect to a line of a curve given in homogeneous, rectangular, line-coördinates is well known. The $r$ th polar of $F(u, v, w)=0$ with respect to the line $\left(u^{\prime}, v^{\prime}, w^{\prime}\right)$ is given, in the usual symbolic notation, by

$$
\left(u^{\prime} \frac{\partial}{\partial u}+v^{\prime} \frac{\partial}{\partial v}+w^{\prime} \frac{\partial}{\partial w}\right)^{r} F=0 .
$$

We propose to extend the name "curve" to a homogeneous rational fractional function of $u, v, w$ (we shall then write curve in italics), and to apply to it the operation of polarization with respect to a line. If $F(u, v, w)$ $\equiv F_{1}(u, v, w) / F_{2}(u, v, w)$, where $F_{1}(u, v, v)$ and $F_{2}(u, v, w)$ are homogeneous polynomials in $u, v, w$, having no common factor that is not a constant, then, as before, the $r$ th polar of the curve $F(u, v, w)=0$ with respect to the line $\left(u^{\prime}, v^{\prime}, w^{\prime}\right)$ is given by

$$
\left(u^{\prime} \frac{\partial}{\partial u}+v^{\prime} \frac{\partial}{\partial v}+w^{\prime} \frac{\partial}{\partial w}\right)^{v} F=0 .
$$

In particular, if the line $\left(u^{\prime}, v^{\prime}, w^{\prime}\right)$ be the line at infinity $\left(u^{\prime}=v^{\prime}=0\right)$, then the $r$ th polar of $F(u, v, w)=0$ is $\frac{\partial r}{\partial w^{r}}\left[F_{1}(u, v, w) / F_{2}(u, v, w)\right]=0$, which we designate, for the sake of brevity, by $F_{w}^{r}=0$. The numerator of $F_{w}^{r *}$ we denote by $\left(F_{w o}^{r}\right)$. With these explanations clearly in mind, we can prove the following lemmas concerning the curve $\left(F_{w}^{r}\right)=0$.

LEMMA 1. If $F_{1}(u, v, w)=0$ touches the $X$-axis in $k_{1}$ points (not necessarily distinct), given by the roots of $\theta_{1}(x)=0$, and if $F_{2}(u, v, w)=0$ touches the $X$-axis in $k_{2}$ distinct and different points given by the roots of $\theta_{2}(x)=0$, the

\footnotetext{
* The numerator and the denominator of $F_{w}^{r}$ have no common factor other than a constant.
} 
points of contact of $\left(F_{v 0}^{*}\right)=0$ with the $X$-axis are given by the roots of the $r$ th derivative of $\theta_{1}(x) / \theta_{2}(x)=0 . *$

Case 1. Suppose $F_{2}(u, v, w)$ has no multiple factors.

Let the roots of $\theta_{1}(x)=0$ and of $\theta_{2}(x)=0$ be $x_{1}, \ldots, x_{k_{1}}$ and $x_{1}^{\prime}, \ldots, x_{k_{2}}^{\prime}$ respectively, where $x_{1}^{\prime} \neq x_{2}^{\prime} \neq \ldots \neq x_{k_{2}}^{\prime}$ and $\theta_{1}\left(x_{1}^{\prime}\right) \neq 0\left(i=1,2, \ldots, k_{2}\right)$. We can write therefore

$$
\theta_{1}(x) \equiv \prod_{i=1}^{k_{1}}\left(x-x_{i}\right)=0, \text { and } \theta_{\mathbf{2}}(x) \equiv \prod_{i=1}^{k_{2}}\left(x-x_{i}^{\prime}\right)=0
$$

If the degree of $F_{1}(u, v, w)$ is $n_{1}$, then, in order that the curve $F_{1}(u, v, v)=0$ should touch the $X$-axis in the points given by

we must have

$$
\prod_{i=1}^{k_{1}}\left(x-x_{i}\right)=0
$$

$$
F_{1}(u, v, w)=v^{n_{1}-k_{1}} \prod_{i=1}^{k_{1}}\left(u x_{i}+w\right)+\boldsymbol{\Phi}_{1}(u, v, w)=0
$$

where the highest power of $v$ in $\Phi_{1}(u, v, w)$ is less than $n_{1}-k_{1}$; for the coordinates of the points of contact of $F_{1}(u, v, w)=0$ with the $X$-axis are the values of $-w / u$ in $F_{\mathrm{g}}(u, v, w)=0$ when $v$ approaches infinity, and these values are the roots of the coefficient of the highest power of $v$ equated to zero when the latter is considered as an equation in $-w / u$. Similarly, if $n_{8}$ is the degree of $F_{2}(u, v, w)=0$, we have

$$
F_{\mathbf{2}}(u, v, w)=v^{n_{2}-k_{2}} \prod_{i=1}^{k_{n}}\left(u x_{i}^{\prime}+w\right)+\boldsymbol{\Phi}_{\mathbf{2}}(u, v, w)=0
$$

where the highest coefficient of $v$ in $\Phi_{2}(u, v, w)$ is less than $n_{2}-k_{2}$. To find the points of contact of $\left(F_{v o}^{r}\right)=0$ with the $X$-axis, we equate the coefficient of the highest power of $v$ in the numerator of $\left(\partial^{r} / \partial w^{r}\right) F(u, v, w)$ to zero. Since

$$
F(u, v, w) \equiv \frac{F_{1}(u, v, v)}{F_{2}(u, v, w)}
$$

* When the derivative has infinite roots arising from the vanishing of the highest coefficient or coefficients of the numerator, homogeneous coördinates should be used. 
we have

$$
\frac{\partial^{r} F}{\partial w^{r}}=F_{1} \frac{\partial^{r}\left(1 / F_{2}\right)}{\partial w^{r}}+r \frac{\partial F_{1}}{\partial w} \frac{\partial^{r-1}\left(1 / F_{2}\right)}{\partial w^{r-1}}+\cdots+\frac{\partial^{r}\left(1 / F_{2}\right)}{\partial w^{r}} .
$$

But we know*

$$
\frac{\partial^{r}\left(1 / F_{2}\right)}{\partial w^{r}}=(-1)^{r} \frac{r !}{F_{2}^{v+1}} \Delta^{r}\left\{F_{\mathbf{2}}\right\}
$$

where

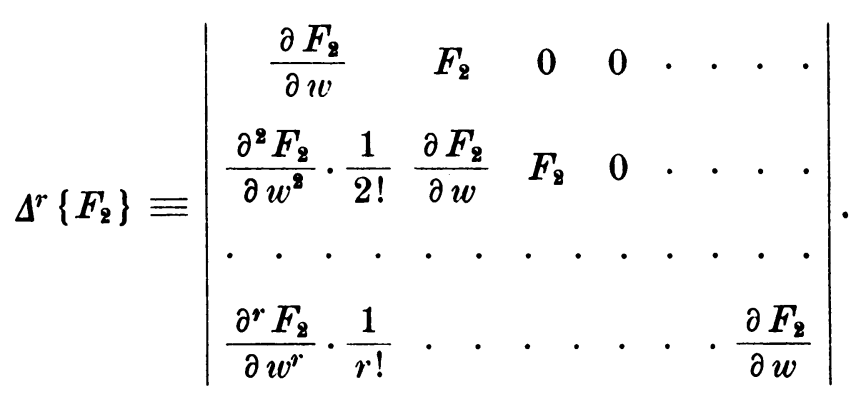

Hence we have the formula

(3) $\quad\left(F_{v v}^{r}\right)=\left[F_{2}\right]^{r} \frac{\partial^{r} F_{1}}{\partial v}-\frac{r !}{(r-1) !}\left[F_{2}\right]^{r-1} \frac{\partial^{r-1} F_{1}}{\partial w^{r-1}} \Delta^{1}\left\{F_{2}\right\}+\cdots$

$$
\cdots+(-1)^{r} r ! F_{1} \Delta^{r}\left\{F_{2}\right\}
$$

as there is no factor common to the expression on the right of equation (3) and to $\left[F_{2}\right]^{r+1}$. If we substitute for $F_{1}$ and $F_{2}$ in the last equation their values given by equations (1) and (2), we see that the coefficient of the highest power of $v$ in this equation, i. e., the coefficient of $v^{n_{1}-k_{1}+r\left(n_{2}-k_{2}\right)}$, is

$$
\prod_{i=1}^{k_{n}}\left(u x_{i}^{\prime}+w\right)^{r} \frac{\partial^{r}}{\partial w^{r}} \prod_{i=1}^{k_{1}}\left(u x_{i}+w\right)
$$

$-\frac{r !}{(r-1) !} \prod_{i=1}^{k_{2}}\left(u x_{i}^{\prime}+w\right)^{r-1} \frac{\partial^{r-1}}{\partial w^{r-1}} \prod_{i=1}^{k_{1}}\left(u x_{i}+w\right)-\Delta^{1}\left\{\prod_{i=1}^{k_{2}}\left(u x_{i}^{\prime}+w\right)\right\}+\cdots$,

* Echols, Calculus, p. 455. 
which is clearly the numerator of

$$
\frac{\partial^{r}}{\partial w^{r}}\left[\prod_{i=1}^{k_{1}}\left(u x_{i}+w\right) / \prod_{i=1}^{k_{s}}\left(u x_{i}^{\prime}+w\right)\right]
$$

The points of contact of $\left(F_{w}^{r}\right)=0$ with the $X$-axis are the roots of the numerator of (5) considered as a function of $-v / u$ and equated to zero. Hence designating $-w / u$ by $x$, we have the points of contact of $\left(F_{w}^{r}\right)=0$ with the $X$-axis given by the roots of the $r$ th derivative of $\theta_{1}(x) / \theta_{2}(x)=0$.

Case 2. $F_{2}(u, v, w)$ has multiple factors.

Since $F_{2}(u, v, w)$ has multiple factors, it is of the form

$$
\begin{aligned}
F_{2}(u, v, w)=\prod_{j=1}^{s}\left[v^{\sigma_{j}}+\right. & \left.\Phi_{\sigma_{j}}(u, v, w)\right]^{t_{j}} \\
& {\left[v^{n_{2}-k_{2}-\sigma} \prod_{i=1}^{k_{s}}\left(u x_{i}^{\prime}+w\right)+\Phi_{n_{2}-\sigma}(u, v, w)\right]^{*} }
\end{aligned}
$$

where $t_{j} \geqq 2, \sigma=\sum_{j=1}^{s} t_{j} \sigma_{j}, n_{2}-k_{2} \geqq \sigma$, and $v^{n_{2}-k_{2}-\sigma} \prod_{i=1}^{k_{2}}\left(u x_{i}^{\prime}+w\right)+\boldsymbol{\Phi}_{n_{2}-\sigma}$ has no multiple factors, for the coefficient of the highest power of $v$ in $F_{2}(u, v, w)$ must be $\prod_{i=1}^{k_{2}}\left(u x_{i}^{\prime}+w\right)$, which has no multiple factors. The expression on the right of equation (3), therefore, has a factor in common with $\left(F_{w}^{r}\right)$, namely

$$
\prod_{j=1}^{s}\left[v^{\sigma_{j}}+\Phi_{\sigma_{j}}(u, v, w)\right]^{r\left(t_{j}-1\right) \dagger}
$$

and $\left(F_{w}^{r}\right)$ is equal to the expression on the right of equation (3) divided by (7). But this does not affect the identity of the coefficients of the highest power of $v$ in $\left(F_{v v}^{r}\right)$ and the numerator of (5). Hence, as before, the points of contact of $\left(F_{v o}^{r}\right)=0$ with the $X$-axis are given by the roots of the $r$ th derivative of $\theta_{1}(x) / \theta_{2}(x)=0$, and the lemma is established.

In the following lemmas we designate $\theta_{1}(x) / \theta_{2}(x)$ by $\theta(x)$, and the $r$ th derivative of $\theta(x)$ by $\theta^{r}(x)$.

* The subscript of $\boldsymbol{D}$ designates its degree.

$\dagger$ The degree of $\left(F_{w}^{r}\right)=0$ is therefore equal to $n_{1}+r\left(n_{2}-\sigma+2 \sum_{j=1}^{s} \sigma_{j}-1\right)$. 
LEMMA $2^{*}$. If not all of the points of contact of $F_{2}(u, v, w)=0$ with the $X$-axis are distinct and different jrom the points of contact of $F_{1}(u, v, w)=0$ with the same axis, i. e.,

$$
\begin{gathered}
\theta_{\mathbf{2}}(x)=\prod_{i=1}^{p}\left(x-x_{i}^{\prime}\right)^{\mu_{i}} \prod_{i=p+1}^{q}\left(x-x_{j}^{\prime}\right)^{\mu_{j}} \prod_{k=q+1}^{s}\left(x-x_{k}^{\prime}\right)=0\left(\mu_{i} \geqq 1, \mu_{j} \geqq 2\right), \\
\theta_{1}\left(x_{i}^{\prime}\right)=0(i=1,2, \ldots, p), \text { and } \theta_{1}\left(x_{i}^{\prime}\right) \neq 0(i=p+1, \ldots, s),
\end{gathered}
$$

and if $F_{\mathrm{z}}(u, v, w)=0$ has multiple factors such that

$$
F_{2}(u, v, w)=\prod_{i=1}^{\tau}\left[v^{\sigma_{i}} \Phi_{\rho_{i}}(u, v)+\Phi_{\sigma_{i}+\rho_{i}}(u, v, w)\right]^{t_{i}} \Phi(u, v, v)=0
$$

where the highest power of $v$ in $\boldsymbol{\Phi}_{\sigma_{i}+\rho_{i}}(u, v, w)$ is less than $\sigma_{i}, t_{i} \geqq 2$, and where $\Phi(u, v, w)$ has no multiple factors, the points of contact of $\left(F_{v}^{v}\right)=0$ with the $X$-axis are given by the roots of

$$
\frac{\left[\prod_{i=1}^{q}\left(x-x_{i}^{\prime}\right)^{\mu_{i}}\right]^{r+1}}{\prod_{i=1}^{\tau}\left[\Phi_{\rho_{i}}(-1, x)\right]^{r\left(t_{i}-1\right)}} \theta^{r}(X)=0, \dagger
$$

provided $\theta^{r}(x) \neq$ 丰 0 .

The proof of this lemma is very similar to the proof of the previous one, and we therefore do not repeat it. We only recall that $\left(F_{w}^{r}\right)$, under the conditions of this lemma, is equal to the expression on the right of equation (3) divided by $\prod_{i=1}^{\tau}\left[v^{\sigma_{i}} \Phi_{\rho_{i}}(u, w)+\Phi_{\sigma_{i}+\rho_{i}}(u, v, w)\right]^{r\left(t_{i}-1\right)}$.

COROLlaRY 1. If $p=0, \tau=q, \sigma_{i}=0, t_{i}=\mu_{i}$, and $\Phi_{\rho_{i}}(u, w)$ $\equiv u x_{i}^{\prime}+w$, then the points of contact of $\left(F_{w}^{r}\right)=0$ with the $X$-axis are again the roots of $\theta^{r}(x)=0$.

LEMMA 3. If the $x$-intercepts of the tangents of $F_{1}(u, v, w)=0$ of slope $m$ are given by the roots of $\theta_{1}(x)=0$, and if the $x$-intercepts of the tangents of $F_{\mathrm{z}}(u, v, w)=0$ of the same slope are given by the roots of $\theta_{\mathrm{a}}(x)=0$ which

* While this lemma is more general than we need, the proof of the less general one is not much simpler.

$\dagger$ An infinite value of the denominator is not here considered a root. 
are distinct and different from the roots of $\theta_{1}(x)=0$, the $x$-intercepts of the tangents of $\left(F_{w}^{r}\right)=0$ of slope $m$ are given by the roots of $\theta^{r}(x)=0$.

LEMMA 4. If not all the tangents of $F_{2}(u, v, w)=0$ of slope $m$ are distinct and different from the tangents of $F_{1}(u, v, w)=0$ of the same slope, i.e.,

$$
\begin{gathered}
\theta_{\mathbf{2}}(x)=\prod_{i=1}^{p}\left(x-x_{i}\right)^{\mu_{i}} \prod_{j=p+1}^{q}\left(x-x_{j}\right)^{\mu_{j}} \prod_{k=q+1}^{s}\left(x-x_{k}\right)=0\left(\mu_{i} \geqq 1, \mu_{j} \geqq 2\right), \\
\theta_{1}\left(x_{i}\right)=0(i=1,2, \ldots, p), \text { and } \theta_{1}\left(x_{i}\right) \neq 0(i=p+1, \ldots, s),
\end{gathered}
$$

and if $F_{\mathbf{2}}(u, v, w)=0$ has multiple factors such that

$$
F_{\mathbf{2}}(u, v, w) \equiv \prod_{i=1}^{\tau}\left[\boldsymbol{\Phi}_{\rho_{i}}(u, w)+v \boldsymbol{\Phi}_{\rho_{i}-1}(u, v, w)\right]^{t_{i}} \boldsymbol{\Phi}(u, v, w)=0
$$

where $\boldsymbol{\Phi}(u, v, w)$ has no multiple factor, and where $t_{i} \geqq 2$, the $x$-intercepts of the tangents of $\left(F_{v}^{r}\right)=0$ with the slope $m$ are given by the roots of

$$
\frac{\left[\prod_{i=1}^{q}\left(x-x_{i}\right)^{\mu_{i}}\right]^{r+1}}{\prod_{i=1}^{\tau}\left[\Phi_{\rho_{i}}(-1, x)\right]^{r\left(t_{i}-1\right)}} \theta^{r}(x)=0,
$$

provided $\theta^{r}(x)$ 丰 0 .

COROLlary 1. If $p=0, \tau=q, t_{i}=\mu_{i}$ and $\Phi_{\rho_{i}}(u, w) \equiv u x_{i}+w$ $(i=1, \ldots, q)$, the $x$-intercepts of the parallel tangents of $\left(F_{v 0}^{r}\right)=0$ are again the roots of $\theta^{r}(x)=0$.

LEMMA 5. If the foci of $F_{1}(u, v, w)=0$ and of $F_{2}(u, v, w)=0$ are given in the complex plane by the roots of the polynomials $f_{1}(z)=0$ and $f_{8}(z)=0^{*}$ respectively, such that the roots of $f_{\mathbf{2}}(z)=0$ are distinct and different from the roots of $f_{1}(z)=0$, the foci of $\left(F_{w}^{r}\right)=0$ are the roots of the rth derivative of $f_{1}(z) i f_{8}(z)=0$.

* The foci of $F_{1}(u, v, w)=0$ and of $F_{2}(u, v, w)=0$ are here assumed to be finite. The extension of this lemma to include the case of infinite foci is made obvious by the introduction of homogeneous complex variables. We have no occasion here to use the extension. The proofs of these lemmas are similar to the proof of Lemma 1 and are omitted. See Emch, Bulletin of the American Mathematical Society, vol. 25 (1917), p. 157. 
LEMMA 6. If not all the foci of $F_{\mathrm{q}}(u, v, w)=0$ are distinct and different from the foci of $F_{1}(u, v, w)=0$, i.e.,

$f_{\mathrm{z}}(z)=\prod_{i=1}^{p}\left(z-z_{i}\right)^{\mu_{i}} \prod_{j=1}^{q}\left(z-z_{j}\right)^{\mu_{j}} \prod_{k=q+1}^{s}\left(z-z_{k}\right)=0 \quad\left(\mu_{i} \geqq 1, \mu_{j} \geqq 2\right)$, $f_{1}\left(z_{i}\right)=0(i=1, \ldots, p), \quad f_{1}\left(z_{i}\right) \neq 0(i=p+1, \ldots, s)$

and if $F_{\mathbf{2}}(u, v, w)=0$ has multiple factors such that

$$
F_{\mathbf{2}}(u, v, w)=\prod_{i=1}^{\tau}\left[\Phi_{\rho_{i}}(u, v, w)\right]^{t} \boldsymbol{\Phi}(u, v, w)=0
$$

where $t_{i} \geqq 2$ and where $\Phi(u, v, w)$ has no multiple factors, the foci of $\left(F_{w 0}^{r}\right)=0$ are the roots of

$$
\frac{\left[\prod_{i=1}^{q}\left(z-z_{i}\right)^{\mu_{i}}\right]^{r+1}}{\prod_{i=1}^{\tau}\left[\Phi_{\rho_{i}}(-1,-i, z)\right]^{r\left(t_{i}-1\right)}} f^{r}(z)=0,
$$

provided $f^{r}(z)$ 丰 0 .

CoRollary 1. If $p=0, \tau=q, t_{i}=\mu_{i}$, and $\Phi_{\rho_{\mathrm{r}}}(-1,-i, z) \equiv z-z_{i}$ $(i=1,2, \ldots, q)$, the foci of $\left(F_{w}^{r}\right)=0$ are again the roots of $f^{r}(z)=0$.

\section{GENERALIZATION OF VAN DEN BERG'S THEOREM}

Let

$$
f(z) \equiv \prod_{i=1}^{n}\left(z-z_{i}\right)^{\mu_{i}} \quad\left(z_{i} \neq z_{j}, \mu_{i} \leqq \mu_{i+1}, i, j=1, \ldots, n-1\right)
$$

be a rational function of $z$. Let $k$ be the number of distinct poles of $f(z)$, i. e. $\mu_{i}<0, i=1, \ldots, k ; \mu_{i}>0, i=k+1, \ldots, n$. We consider the curve

$$
\varphi(u, v, w) \equiv \prod_{i=1}^{n} \alpha_{i}^{\mu_{1}}=0 \quad\left(z_{i}: \alpha_{i} \equiv u x_{i}+v y_{i}+w\right)
$$


Clearly it is of the type $F(u, v, w)=0$ considered above, where

$$
F_{1}(u, v, w) \equiv \prod_{i=k+1}^{n} \alpha_{i}^{\mu_{i}}=0, \text { and } F_{2}(u, v, w) \equiv \prod_{i=1}^{k} \alpha_{i}^{\left|\mu_{i}\right|}=0
$$

The foci of $\prod_{i=k+1}^{n} \alpha_{i}^{\mu_{i}}=0$ are the roots of $\prod_{i=k+1}^{n}\left(z-z_{i}\right)^{\mu_{i}}=0$, those of $\prod_{i=1}^{k} \alpha_{i}^{\left|\mu_{i}\right|}=0$ are the roots of $\prod_{i=1}^{k}\left(z-z_{i}\right)^{\left|\mu_{i}\right|}=0$, and those of $\left(\varphi_{v v}^{r}\right)=0$ are given by Corollary 1 , Lemma 4. Any line through $\alpha_{i}=0(i \leqq k)$ is tangent to $\prod_{i=1}^{k} \alpha_{i}^{\left|\mu_{i}\right|}=0$ at the point $z_{i}$. Similarly any line through $\alpha_{j}=0(k<j \leqq n)$ is tangent to $\prod_{i=k+1}^{n} \alpha_{i}^{\mu_{i}}=0$ at the point $z_{j}$. If $m_{1}$ of the $n-k$ zeros are collinear with $m_{2}$ of the $k$ poles, and if no other zero of $f(z)$ is collinear with them, then the points of contact of $\left(\varphi_{w}^{r}\right)=0$ with that line are given by Corollary 1, Lemma 2. Hence:

THEOREM 1. The zeros of the rth derivative of the rational function

$$
f(z) \equiv \prod_{i=1}^{n}\left(z-z_{i}\right)^{\mu_{i}}
$$

are the foci of $\left(\varphi_{w}^{r}\right)=0$, where

$$
\varphi(u, v, w) \equiv \prod_{i=1}^{n} \alpha_{i}^{\mu_{i}} \quad\left(z_{i}: \alpha_{i} \equiv u x_{i}+v y_{i}+v\right)
$$

The curve $\left(\varphi_{v o}^{r}\right)=0$ touches the line through m zeros and poles of $f(z)(m<v) *$ in points which are independent of the remaining zeros and poles of $f(z)$, provided no other zero or pole of $f(z)$ lies on that line. If this line be taken for the $X$-axis, the points of contact are given by the roots of the rth derivative of

$$
\prod_{i=1}^{m}\left(x-x_{i}\right)^{\mu_{i}}=0
$$

* When $m=n$ the curve $\left(\varphi_{w}^{r}\right)=0$ degenerates into real and imaginary points. In all further considerations it will be assumed that not all of the zeros and poles of $f(z)$ are collinear. 
where $x_{1}, x_{2}, \ldots, x_{m}$ are the zeros and poles that lie on that line, and where $\mu_{1}, \mu_{2}, \ldots, \mu_{m}$ are their multiplicities in $f(z)$.

Van den Berg's theorem follows from this when the function $f(z)$ is an integral rational function and $r=1$, except that (as van den Berg and also Heawood failed to point out) the point of contact on a segment $z_{i} z_{j}$ does not divide the segment in the ratio of $\mu_{i}$ to $\mu_{j}$ in case some other zero of $f(z)$ lies on that same segment.

In case $\mu_{i}>r$, the curve $\left(\varphi_{v}^{v}\right)=0$ will have a factor $\alpha_{i}^{\mu_{i}-r}$. We define $\left[\varphi_{w}^{r}\right]=0$ to be that part of $\left(\varphi_{w}^{r}\right)=0$ remaining after all the factors $\prod_{i=l+1}^{n} \alpha_{i}^{\mu_{i}-r}$ $\left(\mu_{i}>r, i=l+1, \ldots, n\right)$ of $\left(\varphi_{w}^{v}\right)=0$ have been suppressed. The curve under investigation is $\left(\varphi_{v v}^{r}\right)=0$, but more particularly $\left[\varphi_{v 0}^{r}\right]=0$. The curve in van den Berg's theorem is $\left[\varphi_{w}^{\prime}\right]=0$. It follows, from the discussion of $\left(F_{v v}^{r}\right)=0, *$ that if $(\nu)$ and $[\nu]$ designate the degrees of $\left(\varphi_{v v}^{r}\right)=0$ and $\left[\varphi_{w}^{r}\right]=0$ respectively,

$(\nu)=r(k-1)+\sum_{i=k+1}^{n} \mu_{i}$, and $[\nu]=r(k-1)+\sum_{i=k+1}^{n} \mu_{i}-\sum_{i=l+1}^{n}\left(\mu_{i}-r\right)$.

\section{POINTS OF CONTACT ON $z_{i} z_{j}$}

Let $z_{i} z_{j}$ be two of the points $z_{1}, z_{2}, \ldots, z_{n}$ such that none of the remaining $n-2$ points lies on the line joining $z_{i}$ to $z_{j}$. When this line $z_{i} z_{j}$ is tangent to $\left(\varphi_{v o}^{r}\right)=0$ and $\left[\varphi_{v w}^{r}\right]$ we shall denote the multiplicity of its tangency by $\left(\nu_{i j}\right)$ and $\left[\nu_{i j}\right]$ respectively. Since the order of a multiple tangent is equal to the number of points of contact on it - distinct or coincident - we can construct the table given below.

It is clear, from this table, that the only case in which $\left(\varphi_{w}^{r}\right)=0$ and $\left[\boldsymbol{s}_{w}^{r}\right]=0$ are not tangent to $z_{i} z_{j}$ is that in which $\mu_{i}>0, \mu_{j}>0$, and $\mu_{i}+\mu_{j} \leqq r$. Furthermore $\left(\nu_{i j}\right)=\left[\nu_{i j}\right]$ except when $\mu_{i}>r$, or when $\mu_{j}>r$, or when both $\mu_{i}$ and $\mu_{j}$ are greater than $r$.

For $\mu_{i}>0$ and $\mu_{j}>0$ the points of contact of $\left[\varphi_{w}^{r}\right]=0$ with the line $z_{i} z_{j}$ are always real and distinct and lie between $z_{i}$ and $z_{j}$. The points of contact of $\left[\varphi_{w o}^{r}\right]=0$ separate the points of contact of $\left[\varphi_{v 0}^{r-1}\right]=0$. If one of the $\mu^{\prime}$ 's, say $\mu_{i}$, is negative and if $\mu_{i}+\mu_{j}<0$, the points of contact with $\left[\varphi_{w}^{v}\right]=0$ are still finite, real, and distinct, but lie now on the extension of $z_{i} z_{j}$. The points of contact of $\left[\varphi_{v}^{r}\right]=0$ and of $\left[\varphi_{v 0}^{r-1}\right]=0$ are mutually separated. If $\mu_{i}+\mu_{j}=0$, the distribution of points of contact is the same as in the case $\mu_{i}+\mu_{j}<0$, with one exception; namely, all but one of the points of con-

* See footnote on p. 243.

$\dagger$ A point is said to lie on the extension of $z_{i} z_{j}$ if $z_{j}$ lies between the point and $z_{i}$. 


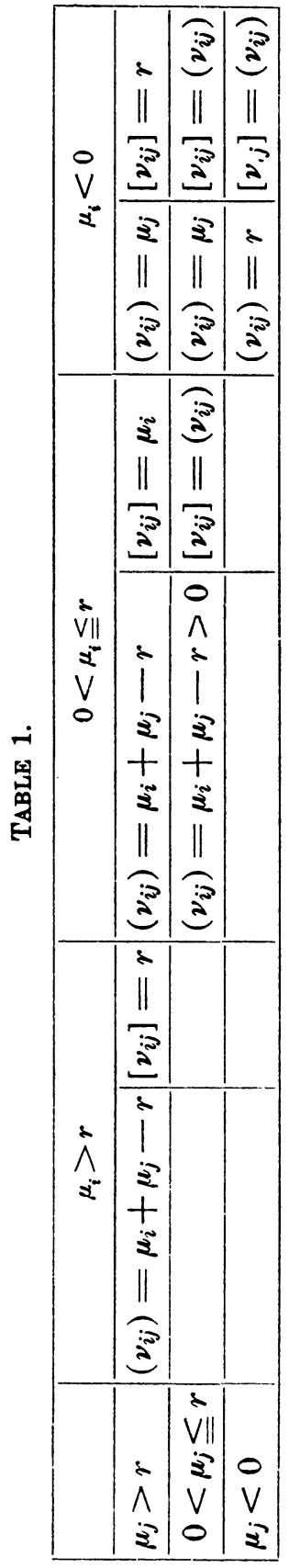

tact are finite. The line through $z_{i} z_{j}$ is therefore an asymptote of $\left[\varphi_{v i}^{r}\right]=0$ with or without other finite points of contact on it, and, as the point of tangency at infinity counts for just one of the points of contact of that line with $\left[\varphi_{v}^{r}\right]=0$, the two asymptotic branches of $\left[\varphi_{v o}^{r}\right]=0$ lie on different sides of $z_{i} z_{j}$. Furthermore, if $m$ zeros are collinear and no pole is collinear with them, then the points of contact of $\left[\varphi_{v i}^{r}\right]=0$ with that line are still finite, real, and distinct. Moreover, if one pole is collinear with $m$ zeros and the order of the pole is greater than the sum of the orders of the zeros, the points of contact with $\left[\varphi_{v 0}^{r}\right]=0$ are still finite, real, and distinct. Finally, if $z_{1}, z_{2}, \ldots, z_{m}$ are collinear, so that $\mu_{i}>0\left(i=1, \ldots, k^{\prime}\right), 0<\mu_{j} \leqq r\left(j=k+1, \ldots, l^{\prime}\right)$, $\mu_{\lambda}>r\left(\lambda=l^{\prime}+1, \ldots, m\right)$, and if $\left(\nu_{m}\right)$ and $\left[\nu_{m}\right]$ designate the multiplicity of this tangent in $\left(\varphi_{v o}^{r}\right)=0$ and $\left[\varphi_{v 0}^{v}\right]=0$ respectively, then

$$
\begin{aligned}
& \left(\nu_{m}\right)=r\left(k^{\prime}-1\right)+\sum_{i=k^{\prime}+1}^{m} \mu_{i}, \\
& \quad \text { and }\left[\nu_{m}\right]=(\nu)-\sum_{i=l^{\prime}+1}^{m}\left(\mu_{i}-r\right) .
\end{aligned}
$$

\section{The tangents FROM $z_{j}$ TO $\left[\varphi_{w}^{r}\right]=0$}

Let the curve $\theta(u, v, w)=0$ be defined by the identity

$$
\varphi(u, v, w) \equiv \boldsymbol{\alpha}_{j}^{\mu_{j}} \theta(u, v, w) \quad(j \leqq n)
$$

Then

$$
\begin{gathered}
\frac{\partial^{r} \varphi(u, v, w)}{\partial w^{r}} \equiv \\
\sum_{\lambda=0}^{r} \frac{r !}{(r-\lambda) !}-\frac{\mu_{j} !}{\left(\mu_{j}-\lambda\right) !} \frac{\alpha_{j}^{\mu_{j}-\lambda}}{\lambda !} \frac{\partial^{r-\lambda} \theta(u, v, w)}{\partial w^{r-\lambda}}
\end{gathered}
$$

and according as (1) $\mu_{j} \geqq r,(2) 0<\mu_{j}<r,(3) \mu_{j}<0$, we have the following three expressions for $\left(\varphi_{v o}^{r}\right)=0$ : 
(i) $\quad\left(\varphi_{v 0}^{r}\right)=\alpha^{\mu_{j}-r} \sum_{\lambda=0}^{r} A_{\lambda} \alpha^{r-\lambda}\left(\theta_{v}^{r-\lambda}\right) \prod_{i=1}^{k} \alpha_{i}^{\lambda}, \quad A_{\lambda}=\frac{r !}{(r-\lambda) !} \frac{\mu_{j} !}{\left(\mu_{j}-\lambda\right) !} \frac{1}{\lambda}$,

(ii) $\quad\left(\varphi_{w}^{r}\right)=\sum_{\lambda=0}^{\mu_{j}} A_{\lambda} \alpha^{\mu_{j}-\lambda}\left(\theta_{w}^{r-\lambda}\right) \prod_{i=1}^{k} \alpha_{i}^{\lambda}$

(iii) $\left(\boldsymbol{\varphi}_{w}^{r}\right)=\sum_{\lambda=0}^{r} A_{\lambda} \boldsymbol{\alpha}^{r-\lambda}\left(\boldsymbol{\theta}^{r-\lambda}\right) \prod_{i=1}^{k} \boldsymbol{\alpha}_{i}^{\lambda} / \boldsymbol{\alpha}_{j}^{\lambda}$.

Also in all three cases the identity $\left(\varphi_{w}^{r}\right) \equiv\left[\varphi_{v}^{r}\right] \cdot \prod_{i=l+1}^{n} \alpha_{i}^{\mu_{i}-r}$ holds.* It follows therefore that

(i) $\left[\varphi_{v i}^{r}\right]=\alpha_{j} \sum_{\lambda=0}^{r-1} A_{\lambda} \Phi_{\lambda}+A_{r} \prod_{i=1}^{k} \alpha_{i}^{r} \cdot \prod_{i=k+1}^{l} \alpha_{i}^{\mu_{i}} \cdot \prod_{i=l+1, i \neq j}^{n} \alpha_{i}^{r}$

$$
\left(\Phi_{\lambda} \equiv \alpha_{j}^{r-\lambda-1}\left(\theta_{v}^{r-\lambda}\right) \prod_{\substack{i=1 \\ i \neq j}}^{k} \alpha_{i}^{\lambda} / \prod_{\substack{i=l+1 \\ i \neq j}}^{n} \alpha_{i}^{\mu_{i}-r}\right)
$$

(ii) $\left[\varphi_{v o}^{r}\right]=\alpha_{j} \sum_{\lambda=0}^{\mu_{j}-1} A_{\lambda} \alpha^{\mu_{j}-r} \Phi_{\lambda}+A_{\mu_{j}} \prod_{i=1}^{k} \alpha_{i}^{\mu_{j}}$

$$
\prod_{i=k+1, \mu_{i}+\mu_{j}>r, i \neq j}^{l} \alpha_{i}^{\mu_{i}+\mu_{j}-r} \cdot \prod_{i=l+1}^{n} \alpha_{i}^{\mu_{j}} \cdot\left[\theta_{w}^{r-\mu_{j}}\right], \dagger
$$

(iii) $\left[\varphi_{w}^{r}\right]=\alpha_{j} \sum_{\lambda=0}^{r-1} A_{\lambda} \Phi_{\lambda}+A_{r} \prod_{i=1, i \neq j}^{k} \alpha_{i}^{r} \cdot \prod_{i=k+1}^{l} \alpha_{i}^{\mu_{i}} \cdot \prod_{i=l+1}^{n} \boldsymbol{\alpha}_{i}^{r}$.

Hence we see again $\ddagger$ that if $\mu_{j} \geqq r$ or if $\mu_{j}<0$, the totality of tangents from $z_{j}$ to $\left[\varphi_{u}^{r}\right]=0$ consists of the lines joining $z_{j}$ to $z_{i}(i=1, \ldots, k ; i=l+1$, $\ldots, n ; i \neq j$ ), the order of the tangents in this case being $r$, and the lines

* The letter $l$ desiguates the number of zeros whose order is less than $r$.

t We define $\left(\theta_{w}^{r-\mu_{j}}\right) \equiv\left[\theta_{w}^{\left.r-\mu_{j}\right]} \prod_{i=k+1, \mu_{i}+\mu_{j}>r}^{n} \alpha^{\mu_{i}+\mu_{j}-r}\right.$ by analogy with $\left[\varphi_{w}^{r}\right]$.

$\ddagger$ We assume here that $z_{j}$ is not collinear with any two of the remaining $z$ 's. In case of collinearity we use equation (8). 
joining $z_{j}$ to $z_{i}(i=k+1, \ldots, l)$, in which case the order of the tangents is $\mu_{j}$. If, on the other hand, $0<\mu_{j}<r$, the totality of tangents from $z_{j}$ to $\left[\varphi_{w}^{r}\right]=0$ consists of (a) the lines joining $z_{j}$ to $z_{i}(i=1, \ldots, k ; i=l+1$, $\ldots, n)$, the order of the tangents in this case being $\mu_{j}$; (b) the lines joining $z_{j}$ to $z_{i}\left(i=k+1, \ldots, l ; i \neq j ; \mu_{i}+\mu_{j}>r\right)$ in which case the order of the tangents is $\mu_{i}+\mu_{j}-r$; and (c) the lines from $z_{j}$ tangent to $\left[\theta_{w}^{r-\mu_{j}}\right]=0$.

In cases (a) and (b) above the points of contact of a tangent through $z_{i} z_{j}$ are given by Theorem 1. We proceed to determine the points of contact in case (c).

For the sake of simplicity let $\alpha_{j}=0$ be the origin $\left(x_{j}=y_{j}=0\right)$. Then for values of $u, v, v$ which satisfy $\alpha_{j}=\left(\theta_{v}^{r-\mu_{j}}\right)=0$, inasmuch as $\left(\theta^{r-\mu_{j}+1}\right)$ $=\frac{\partial\left(\theta^{r-\mu_{j}}\right)}{\partial w} \prod_{i=1}^{k} \alpha_{i}$, we have these three relations:

$$
\begin{gathered}
\frac{\partial\left(\varphi_{w}^{r}\right)}{\partial u}=\frac{r !}{\left(r-\mu_{j}\right) !} \frac{\partial\left(\theta^{r-\mu_{j}}\right)}{\partial u} \prod_{i=1}^{k} \alpha_{i}^{\mu_{j}}, \\
\frac{\partial\left(\varphi_{w}^{r}\right)}{\partial v}=\frac{r !}{\left(r-\mu_{j}\right) !} \frac{\partial\left(\theta_{v}^{r-\mu_{j}}\right)}{\partial v} \prod_{i=1}^{k} \alpha_{i}^{\mu_{j}}, \frac{\partial\left(\varphi_{v}^{r}\right)}{\partial w}=\frac{(r+1) r !}{\left(r-\mu_{j}+1\right) !} \cdot \prod_{i=1}^{k} \alpha_{i}^{\mu_{j}}
\end{gathered}
$$

Now making use of the fact that the coördinates of the point of contact on a tangent $\left(u^{\prime}, v^{\prime}, w^{\prime}\right)$ to the curve $\psi(u, v, w)=0$ are given by

we get

$$
-\frac{X}{\frac{\partial \psi}{\partial u^{\prime}}}=\frac{Y}{\frac{\partial \psi}{\partial v^{\prime}}}=\frac{1}{-\frac{\partial \psi}{\partial w^{\prime}}},
$$

$$
X_{\left(\varphi_{10}^{r}\right)}=\frac{r-\mu_{j}+1}{r+1} \cdot X_{\left(\theta^{\left.r-\mu_{j}\right)}\right.}, \text { and } Y_{\left(\varphi_{w}^{r}\right)}=\frac{r-\mu_{j}+1}{r+1} \cdot Y_{\left(\theta_{w}^{r-\mu_{j}}\right)},
$$

provided the tangent under consideration is not singular. Hence,

THEOREM 2. When $\mu_{j} \geqq r$ or $\mu_{j}<0(j \leqq n)$, all the tangents from $z_{j}$ to $\left[\varphi_{v 0}^{r}\right]=0$ pass through the remaining zeros and poles of $f(z)$. When $0<\mu_{j}<v$ $(j \leqq n)$, all the tangents from $z_{j}$ either $(a)$ pass through the poles of $f(z)$, or those zeros of $f(z)$ whose order added to $\mu_{j}$ is greater than $r$, or $(b)$ are tangent to $\left[\theta_{v o}^{r-\mu_{j}}\right]=0$, where $\theta(u, v, w) \equiv \varphi(u, v, w) / \alpha_{j}^{\mu_{j}}$. The point of contact on a tangent to $\left[\theta_{w}^{r-\mu_{j}}\right]=0$ divides the segment from $z_{j}$ to the point of contact with $\left[\theta^{r-\mu_{j}}\right]=0$ in the ratio of $r-\mu_{j}+1$ to $\mu_{j}$, provided that the last tangent is not singular. 
While the equation of the tangents from a general point is not very illuminating, the equation of the tangents from any point at infinity is instructive. We consider this case next.*

\section{PaRAllel tangents OF $\left(\varphi_{v o}^{r}\right)=0$}

THEOREM 3. The tangents of $\left(\varphi_{w}^{v}\right)=0$ parallel to any direction remain invariant if each of the points $z_{1}, z_{2}, \ldots, z_{n}$ is translated parallel to that divection an arbitrary distance.t If a line perpendicular to the direction of the tangents be taken for the $X$-axis and if $x_{i} \neq x_{j}(i, j=1,2, \ldots, n)$, the equation of the vertical tangents is given by the rth derivative of

$$
\prod_{i=1}^{n}\left(x-x_{i}\right)^{\mu_{i}}=0
$$

The proof of this theorem follows immediately from Corollary 1, Lemma 4. If the projection of a pole of $f(z)$ on the $X$-axis coincides with the projection of another pole, or with the projection of a root of the same function on the same axis, the vertical tangents of $\left(\varphi_{w}^{r}\right)=0$ are given by the $r$ th derivative of $\prod_{i=1}^{n}\left(x-x_{i}\right)^{\mu_{i}}=0$ multiplied by a factor which is determined by Lemma 4 . The factor does not add new distinct tangents, but merely increases the multiplicity of the old ones.

We can imagine the curve $\left(\varphi_{w}^{r}\right)=0$ to be the envelope of its parallel tangents, and our investigation of its properties will, in large part, be based on a study of the distribution of its parallel tangents, and the distribution of the points of contact on the tangents through the zeros and poles of $f(z)$. Before we proceed to investigate the general curve $\left(\varphi_{v}^{v}\right)=0$ we apply the above theorems to the curves $\left[y_{i v}^{r}\right]=0$ of class two, i. e., to the conics.

\section{APPLICATIONS; $[\nu]=2$}

The degree of $\left[\varphi_{v}^{*}\right]=0$ is two when $f(z)$ is of the type

(a) $f_{1}(z)=\left(z-z_{1}\right)^{\mu}\left(z-z_{2}\right)\left(z-z_{3}\right)(\mu>0, r=1, \ldots, \mu ; \mu<0, r=1,2, \ldots)$,

(b) $f_{2}(z)=\left(z-z_{1}\right)^{\mu_{1}}\left(z-z_{2}\right)^{\mu_{2}}\left(z-z_{3}\right)^{\mu_{8}}$ $(r=1)$,

(c) $f_{3}(z)=\prod_{i=1}^{n}\left(z-z_{i}\right)^{\mu_{i}}$

$$
\left(\mu_{i}>0, r=\sum_{i=1}^{n} \mu_{i}-2\right) \text {. }
$$

* The writer wishes to express his obligation to Professor W. C. Graustein, of Harvard University, for certain valuable suggestions he has made concerning the subject matter treated in the two preceding sections.

$\dagger$ Provided $\theta^{r}(x)$ ₹ 0 ; see Lemma 4. 
We propose to study each of those cases in detail and to show how the foci of $\left[\varphi_{i}^{r}\right]=0^{*}$ can be constructed by means of ruler and compass.

TYPE (a) $f(z)=\left(z-z_{1}\right)^{\mu}\left(z-z_{2}\right)\left(z-z_{3}\right)$. The point of contact of $\left\lfloor\varphi_{1}^{r}\right]=0$ with $z_{1} z_{2}$ divides the segment $z_{1} z_{2}$ in the ratio of $\mu-r+1$ to $r$, internally or externally according as $\mu \gtrless 0$. Similarly the point of contact with $z_{1} z_{3}$ divides the segment $z_{1} z_{3}$ in the same ratio. Let $B$ and $C$ be the points of contact on $z_{1} z_{2}$ and $z_{1} z_{3}$ respectively; then $B C$ is parallel to $z_{5} z_{3}$ and therefore the center of the conic $\left[y_{1}^{r}\right]=0$ lies on that median of the triangle $z_{1} z_{2} z_{3}$ which is drawn from $z_{1}$. The tangent parallel to $z_{1} z_{3}$ intersects $z_{1} z_{2}$ in a point which divides $z_{1} z_{2}$ in the ratio of $\mu-r+2$ to $r$, internally or externally according as $\mu \gtrless 0$. Also the tangent parallel to $z_{1} z_{2}$ divides $z_{1} z_{3}$ in the same ratio. Hence the center of the conic divides the median in the ratio of $\mu-r+2$ to $r$. Furthermore, when $\mu>0$ the conic is always an ellipse; when $\mu<0$, but $\mu+1 \neq 0$ the conic is an ellipse, a parabola, or a hyperbola according as $\mu+2 \lesseqgtr 0$; and finally, when $\mu+1=0$ the conic is a hyperbola, for $r=1$, but degenerates into two pencils of lines parallel to $z_{1} z_{2}$ and $z_{1} z_{3}$ for all other values of $r$. These results follow at once from the fact that a conic is an ellipse or a hyperbola according as it passes through a point between or not between two parallel tangents, while it is a parabola if it touches the line atinfinity.

To construct the foci of $\left[\varphi_{1}^{r}\right]=0$ by means of a ruler and a compass we make use of the two following theorems:t The conjugate diameters of a central conic set up on a circle through the center of the conic an involution whose rectangular pair are the axes of the conic; and a tangent and a normal at any point of a central conic intersect the minor axis of the conic in points which are concyclic with the foci. the center of $\left[\varphi_{1}^{r}\right]=0$, draw $O E$ and $O F$ parallel to $z_{1} B$ and $z_{1} C$ respectively. Then $O B, O E$ and $O C, O F$ are two pairs of conjugate directions of the last conic. Now take any point $O^{\prime}$ as a center and draw a circle through $O$. Let this circle intersect $O B, O E, O C$, and $O F$ in $B^{\prime}, E^{\prime}, C^{\prime}$ and $F^{\prime}$ respectively. Denote by $D$ the intersection of $B^{\prime} E^{\prime}$ and $C^{\prime} F^{\prime}$, and let $O D$ intersect the circle in the points $M$ and $N$. Then $O M$ and $O N$ are in the direction of the axes of $\left[\varphi_{1}^{r}\right]=0$. Let $z_{1} B$ and the perpendicular to $z_{1} B$ at $B$ intersect the minor axis\& in $P$ and $Q$ respectively. Then the circle on $P Q$ as a diameter intersects the major axis in the foci of $\left[\varphi_{1}^{r}\right]=0 . \|$

${ }^{*}\left[\varphi_{i}^{r}\right]=0$ is the $\varphi$ curve corresponding to $f_{i}(z) \quad(i=1,2,3)$.

$\dagger$ This method of constructing the axes of a conic was called to my attention by Professor C. M. Sparrow, of the University of Virginia.

$\ddagger$ Casey, Analytical Geometry, 2d edition, p. 215.

$\S$ The normal at any point (not a vertex) of a central conic first intersects the major axis and then the minor axis.

II In the case of a cubic with simple roots compare the above method with one given by J. L. Walsh, Annals of Mathematics, vol. 22 (1920), p. 142. 
The construction is much simplified if the triangle $z_{1} z_{2} z_{3}$ is isosceles, $z_{1} z_{2}=z_{1} z_{3}$. Then the median through $z_{1}$ and the perpendicular to it at $O$ are the axes of $\left[\varphi_{1}^{r}\right]=0$, and, as before, $z_{1} B$ and the normal at $B$ determine a circle which intersects the major axis in the foci. If we take the above median for the axis of reals, the roots of $f_{1}^{r}(z)=0$ are real or imaginary according as the intersection of the normal at $B$ and the axis of reals lies inside or outside the segment $z_{1} O$. Furthermore, if the normal at $B$ passes through $O$, the roots of $f_{1}^{r}(z)=0$ are coincident and $\left[\varphi_{1}^{r}\right]=0$ is a circle.

In case $\left[\varphi_{1}^{r}\right]=0$ is a parabola $(\mu=2)$, the construction is also quite simple. We have merely to make use of the fact that the tangent at any point of a parabola bisects the angle between the line joining that point to the focus and the perpendicular to the directrix from that point. In our case, of course, the directrix is perpendicular to the median through $z_{1}$. Hence we have the construction: Draw the perpendicular bisectors of $z_{1} B$ and $z_{1} C$, and let them intersect the median through $z_{1}$ in the points $E$ and $F$ respectively. Then the intersection of $B E$ and $C F$ is the desired focus.*

TYPE (b) $f_{2}(z)=\left(z-z_{1}\right)^{\mu_{1}}\left(z-z_{2}\right)^{\mu_{2}}\left(z-z_{3}\right)^{\mu_{3}}$. When the powers $(\mu)$ are all positive or all negative, the conic $\left[\varphi_{2}^{\prime}\right]=0$ is an ellipse. If one of the powers is negative it is an ellipse, parabola, or hyperbola according as $\mu_{1}+\mu_{2}+\mu_{3} \lesseqgtr 0$. If two of the powers are negative it is an ellipse, a parabola, or a hyperbola according as $\mu_{1}+\mu_{2}+\mu_{3} \gtreqless 0$. When the conic is central its center is the center of gravity of the three masses (negative or positive) $\mu_{2}+\mu_{3}, \mu_{3}+\mu_{1}, \mu_{1}+\mu_{2}$ considered located at the vertices of the triangle $z_{1} z_{2} z_{3}$ in the order designated. If the conic is a parabola, the signs of all three powers can not be the same. Let $\mu_{2}$ and $\mu_{3}$ be of like sign. Then the line joining $z_{1}$ to the midpoint of the join of the point of contact on $z_{1} z_{2}$ and the point of contact on $z_{1} z_{3}$ is perpendicular to the directrix of the parabola. Having the center of a central conic or the direction of the directrix of a parabola in addition to the points of contact on two tangents, we can construct the foci or focus by means of ruler and compass as in case $(a)$.

Again the construction of the foci may be considerably simplified for particular functions $f_{\mathbf{z}}(z)$. We mention only two such cases here. If $\mu_{1}+\mu_{2}=0$, the side $z_{1} z_{2}$ is an asymptote, and the center divides the side $z_{1} z_{2}$ in the ratio of $\mu_{2}+\mu_{3}$ to $\mu_{1}+\mu_{3}$. The fourth harmonic of $O C$, OF and $O z_{1}$, where $O$ is the center, $C$ the point of contact on $z_{1} z_{3}$, and $O F$ a line parallel to $z_{1} z_{3}$, is the second asymptote. If also $\mu_{1}+\mu_{3}=0$, then $z_{1} z_{3}$ is the second asymptote. In either case, the bisectors of the angles between the asymptotes are the axes, and the tangent $z_{2} z_{3}$, together with the normal at

\footnotetext{
*It follows therefore that the focus of $\left[\varphi_{1}^{r}\right]=0$ lies on the symmedian through $z_{1}$ for all values of $r$, and that the distance between two successive foci is constant.
} 
the point of contact on it, determines a circle which intersects the major axis in the foci.

TYPE (c) $f_{3}(z)=\prod_{i=1}^{n}\left(z-z_{i}\right)^{\mu_{i}}$. By means of a repeated application of Theorem 2 we can construct the tangents from $z_{i}$ to $\left[\varphi_{3}^{r}\right]=0$ with their points of contact. The remaining part of the construction of the foci of $\left[\varphi_{3}^{*}\right]=0$ is the same as that of $\left[\varphi_{1}^{r}\right]=0 . *$

7. THE POSITION OF THE CURVE $\left[\varphi_{w}^{r}\right]=0$ WITH RESPECT TO THE ZEROS AND POLES OF $f(z)$

Case 1. $f(z)$ is a rational integral function of $z$.

When $r=1$ we have

$$
\left[\varphi_{w}^{1}\right] \equiv \sum_{j=1}^{n} \mu_{j} \frac{\partial \prod_{i=1}^{n} \alpha_{i}}{\partial \alpha_{j}}=0
$$

whose vertical tangents are given by

$$
\sum_{j=1}^{n} \mu_{j} \frac{\partial \prod_{i=1}^{n}\left(x-x_{i}\right)}{\partial\left(x-x_{j}\right)}=0 .
$$

It follows from Rolle's theorem on the roots of the derivative of a polynomial, that, if $x_{i} \neq x_{j}(i, j=1, \ldots, n)$, there is one and only one root of $f(z)$ between any two successive vertical tangents of $\left[\varphi_{w 0}^{r}\right]=0$, and that there are always two roots such that no tangent lies outside of them. If in projecting the roots on some line - say the $X$-axis - it should happen that $x_{i}$ and $x_{j}$ coincide, the perpendicular to the line at $x_{i}$ is a tangent to $\left[\varphi_{v}^{1}\right]=0$. Moreover, if the projections of $m$ zeros coincide, the perpendicular at that point is a tangent of order $m-1$ with $m-1$ finite and distinct points of contact on it. If no three roots of $f(z)$ are collinear, the curve $\left[\varphi_{w}^{1}\right]=0$ has always $n-1$ finite and distinct tangents parallel to any direction and therefore has no singularities whatsoever.t On the other hand, if three or more

* For lack of space all details of this construction are omitted. However, it should be noticed that in case all the $z$ 's of $f_{i}(z)$ are collinear the displacement of one of the $z$ 's vertically and the construction of the vertical tangents of the $\left[\varphi_{i}^{r}\right]=0$ corresponding to the new configuration of the $z$ 's gives the roots of $f_{i}^{r}(z)=0$ by Theorem 3 .

$\dagger$ By singularities in a class curve we mean multiple tangents, or inflexions, or combinations of these. 
of the roots of $f(z)$ are collinear, the curve $\left[\varphi_{v}^{1}\right]=0$ has multiple tangents, but still has no inflexions, for the points of contact on such a multiple tangent are finite and distinct. Nor has it any asymptotes, for the number of tangents parallel to an asymptotic direction cannot exceed $n-2$ in a curve of class $n-1$, since the point at infinity is on the curve. It has no infinite branches, for all its tangents are at a finite distance from the origin.

The extension to $\left[\varphi_{v v}^{r}\right]=0$ follows without difficulty. There is one and only one tangent of $\left[\varphi_{r o}^{r-1}\right]=0$ parallel to and between any two successive parallel tangents of $\left[\varphi_{v v}^{r}\right]=0$, and there are two tangents of $\left[\varphi_{v o}^{r-1}\right]=0$ such that no tangent of $\left[\varphi_{r}^{r}\right]=0$ lies outside of them. If no three zeros of $f(z)$ are collinear, $\left[\varphi_{w}^{v}\right]=0$ has always

$$
\sum_{i=1}^{n} \mu_{i}-r-\sum_{\substack{i=1 \\ \mu_{i}>r}}^{n}\left(\mu_{i}-r\right)
$$

finite and distinct tangents parallel to any direction, and therefore has no singularities whatsoever. Furthermore, if three or more of the zeros of $f(z)$ are collinear, $\left[\varphi_{v 0}^{r}\right]=0$ may have multiple tangents, but still can have no inflexions. Finally, $\left[\varphi_{v o}^{r}\right]=0$ has no asymptotes and does not touch the line at infinity. Hence $\left[\varphi_{x}^{r}\right]=0$ is a closed curve.

If the tangents to $\left[\varphi_{v 0}^{r}\right]=0$ from some point are all real, and if this point be considered to vary, then the tangents from it to $\left[\varphi_{v v}^{v}\right]=0$ will remain real as long as it does not cross the curve. For the curve $\left[\varphi_{v 0}^{r}\right]=0$ has no asymptotes and no inflexions. Moreover, as this point crosses the curve it gains two tangents or loses two tangents according as it crosses from the concave or convex side of the curve. But as all the tangents from this point are real it can not gain any tangents; and, therefore, in crossing the curve, it meets the convex side of the curve first. Furthermore, from the point at infinity all the tangents are real. Consequently all the tangents to the curve $\left[\varphi_{w 0}^{r}\right]=0$ drawn from any point outside of it are real, and hence, not only is $\left[\varphi_{w}^{r}\right]=0$ closed, but its external contour is convex toward all points outside of it.

THEOREM 4. The curve $\left[\varphi_{w}^{r}\right]=0$ is closed, has no inflexions, and has no multiple tangents except perhaps through collinear zeros of $f(z) .{ }^{*}$ It lies between the extreme tangents parallel to any direction, and it also lies between the extreme tangents drawn from any one of the zeros of $f(z)$ which lies on the least

* To determine whether or not a line through collinear zeros is a multiple tangent use equation (8). 
rectilinear polygon containing all the zeros of $f(z) .{ }^{*}$ It is convex toward all zeros of $f(z)$ whose order is not less than $r$.

Case 2. $f(z)$ is a rational fractional function of $z$.

If $f(z)$ has one single pole whose order is greater than the sum of the orders of the zeros, all the tangents of $\left[\varphi_{w}^{r}\right]=0$ parallel to any direction are still real. Furthermore, $\left[\varphi_{v v}^{r}\right]=0$ still has no multiple tangents except perhaps through the pole and through a zero or perhaps through two or more collinear zeros. The points of contact on such a multiple tangent are always finite, real, and distinct. Hence $\left[\varphi_{v 0}^{r}\right]=0$ has no inflexions. Continuing this reasoning as we did in the case when $f(z)$ was a rational function, we finally conclude that $\left[\varphi_{v v}^{r}\right]=0$ is closed, that its external contour is convex toward all points lying outside of it, and that it lies between the extreme tangents parallel to any direction.t

When $f(z)$ has more than one pole or when the order of the single pole is not greater than the sum of the orders of its zeros, these conclusions do not generally hold. Even then we can usually determine pairs of slopes such that, for all tangents whose slopes lie between these limiting values, most of the conditions mentioned above will hold.

\section{Generalization OF THE GaUSS'-POLYGON THEOREM}

We are now in a position to generalize the Gauss'-polygon theorem very simply.

The foci of a curve are the intersections of the tangents to the curve from the circular points at infinity. Consequently, at least two of the tangents from a focus are imaginary. Therefore the foci of $\left[\varphi_{v 0}^{r}\right]=0$, when $f(z)$ is a rational integral function, lie between the extreme tangents of $\left[\varphi_{v 0}^{r}\right]=0$ parallel to. any direction. Hence,

THEOREM 5. If the $x$-intercepts of lines through the zeros of a rational integral function, $f(z)$, parallel to any direction, be considered as the zeros of a new real integral function, then those zeros of the rth derivative of $f(z)$ that are distinct from the zeros of $f(z)$ lie between the two lines parallel to the same direction and passing through the least and greatest zero of the rth derivative of the new rational integral function. $\neq$

\footnotetext{
* By considering tangents parallel to this polygon we conclude that $\left[\varphi_{w}^{*}\right]=0$ lies inside this polygon.

$\dagger$ Also the tangents from any point outside of it are all real.

$\ddagger$ This theorem may also be stated in the form suggested by Professor Kempner, of the University of Illinois: Let the zeros of a polynomial $f(z)$ be $x_{1}+i y_{1}, \ldots, x_{n}+i y_{n}$, and let the zeros of $f^{r}(z)$ be $x_{1}^{\prime}+i y_{1}^{\prime}, \ldots, x_{m}^{\prime}+i y_{m}^{\prime}$, where $x_{1}^{\prime} \leqq x_{s}^{\prime} \leqq \cdots \leqq x_{m}^{\prime}$. If now $\bar{x}_{1}$ and $\bar{x}_{m}$ be the least and greatest (real) root of the $r$ th derivative of $g(x) \equiv\left(x-x_{1}\right)\left(x-x_{2}\right) \ldots$ $\left(x-x_{n}\right)$, then $\bar{x}_{1}<x_{1}^{\prime}$ and $\bar{x}_{m}>x_{m}^{\prime}$.
} 
The Gauss'-polygon theorem follows from this when $r=1$ and the directions in the theorem are taken parallel to the sides of the least rectilinear polygon containing all the roots of $f(z)$.

Finally, Theorem 5 applies not only to a rational integral function as stated, but to any rational fractional function whose corresponding curve $\left[\varphi_{v 0}^{r}\right]=0$ is closed, has no inflexions, and all of whose tangents from any point at infinity are real.* The theorem is therefore applicable to a rational function of one multiple pole whose order is greater than the sum of the orders of the zeros.t

HAVARD UNIVERSITY, Cambridge, Mass.

* Cf. Bôcher, Proceedings of the American Academy of Arts and Sciences, vol. 40 (1904), p. 478. Also Walsh, these Transactions, vol. 19 (1918), p. 297.

$\dagger$ Part II of this paper, which deals primarily with the tracing of the $\varphi$-curves, will appear separately. 